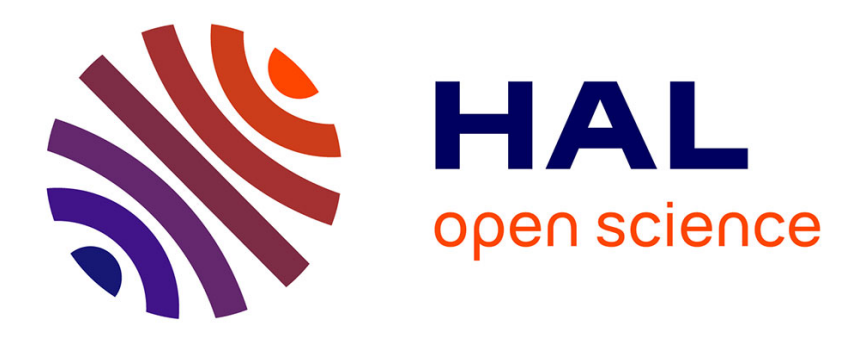

\title{
A simulation of digital image restoration in electron microscopy
}

\author{
H.C. Benski, C. Sonrel
}

\section{To cite this version:}

H.C. Benski, C. Sonrel. A simulation of digital image restoration in electron microscopy. Revue de Physique Appliquée, 1977, 12 (4), pp.543-546. 10.1051/rphysap:01977001204054300 . jpa-00244207

\section{HAL Id: jpa-00244207 https://hal.science/jpa-00244207}

Submitted on 1 Jan 1977

HAL is a multi-disciplinary open access archive for the deposit and dissemination of scientific research documents, whether they are published or not. The documents may come from teaching and research institutions in France or abroad, or from public or private research centers.
L'archive ouverte pluridisciplinaire HAL, est destinée au dépôt et à la diffusion de documents scientifiques de niveau recherche, publiés ou non, émanant des établissements d'enseignement et de recherche français ou étrangers, des laboratoires publics ou privés. 
Classification

Physics Abstracts

$0.691-0.693$

\title{
A SIMULATION OF DIGITAL IMAGE RESTORATION IN ELECTRON MICROSCOPY $\left({ }^{*}\right)$
}

\author{
H. C. BENSKI and C. SONREL
}

Centre d'Etudes Nucléaires de Grenoble, Laboratoire d'Electronique et de Technologie de l'Informatique, Laboratoire Mesure, Contrôle et Traitement Electronique 85 X, 38041 Grenoble Cedex, France

(Reçu le 16 septembre 1976, révisé le 9 décembre 1976, accepté le 21 décembre 1976)

Résumé. - On a simulé une restauration d'image en utilisant un système de traitement du signal équipé d'un minicalculateur pour imiter les conditions du microscope électronique sous le régime d'objets à faible phase. Une correction par filtrage de Wiener est présentée.

\begin{abstract}
An image restoration simulation has been performed using a minicomputer signal processing system to simulate an electron microscope under weak phase object conditions. A Wiener filter correction is presented.
\end{abstract}

1. Introduction. - Recent work in image restoration, in particular for images of high resolution electron microscopy, has used different types of holographic filters $[1,2]$ equivalent to the widely known Wiener filters. We believe that an image processing simulation is desirable for two reasons. In the first place it would produce a case where an original or reference image would be available for comparison, with the degraded image as well as with the restored image. This is missing in real world electron microscopy. Secondly it would provide a comparison of numerical and optical Wiener filters for image processing, despite the fact that, in this case, Wiener filtering might not produce the optimum effect result.

2. Image blurring in electron microscopy. - Weak phase objects are, by definition, considered to produce a small distortion of the phase of the incoming plane wave of the electron beam. The amplitude produced by low contrast objects can be written as :

$$
\exp \{i \varphi(x, y)\} \simeq 1+i \varphi(x, y)
$$

where $\varphi(x, y)$ is a real function. Therefore the field at the entrance pupil of the electron microscope is :

$$
F(u, v)=\delta(u, v)+i \Phi(u, v)
$$

where $u$ and $v$ represent the spatial frequencies corresponding to $x$ and $y$ respectively.

( $^{*}$ This work was performed under the auspices of the Centre National de la Recherche Scientifique, grant number ATP-T24 (1974).
The transmittance of the electron microscope objective can be taken into account by writing the amplitude at the exit pupil as :

$$
F^{\prime}(u, v)=\delta(u, v)+i \Phi(u, v) \exp \{i k W(u, v)\}
$$

where $k=2 \pi / \lambda$ and $W(u, v)$ is the wave aberration due to defocusing and aberrations.

The image recorded at the output of the electron microscope is proportional to the square of the Fourier transform of equation (3). To first order in $\Phi$ :

$$
\begin{aligned}
i^{\prime}\left(x^{\prime}, y^{\prime}\right) & =\left|f^{\prime}\left(x^{\prime}, y^{\prime}\right)\right|^{2} \\
& =1-2 \varphi\left(x^{\prime}, y^{\prime}\right) * \operatorname{Im} T\left(x^{\prime}, y^{\prime}\right)
\end{aligned}
$$

where $f^{\prime}\left(x^{\prime}, y^{\prime}\right)$ and $T\left(x^{\prime}, y^{\prime}\right)$ are the Fourier transforms of $F^{\prime}(u, v)$ and $\exp \{i k W(u, v)\}$ respectively, the prime coordinates indicate the coordinates of the image plane and the symbol $*$ is used to denote a convolution. In the spatial frequency domain one can write equation (4) as :

$$
I(u, v)=\delta(u, v)-2 \Phi(u, v) K(u, v)
$$

where $I(u, v)$ and $K(u, v)$ are the Fourier transforms of $i^{\prime}\left(x^{\prime}, y^{\prime}\right)$ and $\operatorname{Im}\left\{T\left(x^{\prime}, y^{\prime}\right)\right\}$ respectively. $K(u, v)$ represents the transfer function of the system due to aberrations and from the definition of $T\left(x^{\prime}, y^{\prime}\right)$ can be written as :

$$
K(u, v)=\sin k W(u, v) .
$$

In the absence of astigmatism it is possible to write $W(u, v)$ as [3] :

$$
W(u, v)=W(f)=-\frac{C_{\mathrm{s}} \lambda^{4} f^{4}}{4}+\frac{\Delta z \lambda^{2} f^{2}}{2}
$$


where $f=\left(u^{2}+v^{2}\right)^{1 / 2}, C_{\mathrm{s}}$ is the spherical aberration coefficient and $\Delta z$ is the defocusing. From equations (6) and (7) we see that $K(f)$ is an oscillating function whose period gets smaller as $f$ gets bigger. This is a most disturbing feature of weak phase electron microscopy since its effect is to produce contrast reversals at neighbouring frequencies which, if left uncorrected, can blur an image beyond recognition.

To complete our description of the transfer function in electron microscopy we should include, besides the aberration factor, the effect of the aperture of the microscope and the influence of partial spatio-temporal coherence of the electron beam. This is done by multiplying the second term of equation (5) by the functions $B(f)$ and $E_{\mathrm{c}}(f)$ where the aperture function $B(f)$ equals 1 inside the aperture and 0 elsewhere and $E_{\mathrm{c}}(f)$ is a rapidly decreasing function of $f$. We have simplified existing models of partial coherence $[4,5]$ for the purpose of our simulation and write :

$$
E_{\mathrm{c}}(f)=\exp \left\{-f^{4} / f_{0}^{4}\right\} \text {. }
$$

We can now write equation (5) as :

$I(u, v)=\delta(u, v)-2 B(f) E_{\mathrm{c}}(f) \sin \left[\frac{2 \pi}{\lambda} W(f)\right] \Phi(u, v)$.

The $\delta(u, v)$ term represents a uniform background and the second term contains all the information relative to the object. The final equation which describes the formation of images of weak phase objects can now be expressed as :

$$
I(u, v)=\delta(u, v)+2 \Phi(u, v) H(f)
$$

where $H(f)$ is the total transfer function.

We will now take into account the possible presence of additive white noise and obtain :

$$
I(u, v)=\delta(u, v)+2 \Phi(u, v) H(f)+N(f) .
$$

We point out that the function $H(f)$ can be experimentally determined by taking the digital or optical Fourier transform of the image of a random, uniform noise object such as an amorphous carbon foil [1].

3. Image restoration by Wiener filtering. - In the case of isoplanatic impulse response and uncorrelated noise the problem of signal retrieval can be stated as follows. We start with a given output signal $I(u, v)$ such that :

$$
I(u, v)=S(u, v) H(f)+N(f)
$$

where $S(u, v)$ is the input signal, $H(f)$ the transfer function of the system and $N(f)$, as above, the noise spectrum. We now produce a filter function $\psi(f)$ such that:

$$
\tilde{I}(u, v)=I(u, v) \psi(u, v)
$$

will give a good estimate $\tilde{I}(u, v)$ of the input signal
$S(u, v)$ according to a given criterion. Since there is no absolute quantitative criterion for best ressemblance of two images, the filtering problem in image restoration seems hopeless. Nevertheless many attempts have been made to generate suitable filter structures which would give subjectively good, deblurred images $[6,7,8]$. One of these filters that has attracted considerable attention in optical filtering is the Least Mean-squared Error or Wiener filter [1, 2, 6]. This filter can be written as:

$$
\psi_{\mathrm{w}}=\frac{H^{\dagger}(f)}{|H(f)|^{2}+\frac{P_{\mathrm{N}}(f)}{P_{\mathrm{S}}(u, v)}}
$$

where $H^{\dagger}(f)$ is the complex conjugate of the transfer function and $P_{\mathrm{N}} / P_{\mathrm{S}}$ is the ratio of the noise and input signal power spectra. This filter minimizes the expectation value of the quantity $|\tilde{I}(u, v)-S(u, v)|^{2}$. Other criteria of best estimate, like power spectrum equalization [8], or constrained least squares [9], will produce correspondingly different filter structures.

It is also possible to take into account the human visual response and incorporate it in the construction of the filter [7]. We have concentrated on the Wiener filter mainly because of the possibility of comparison with holographic deblurring techniques that have precisely used this type of filtering in the context of electron microscopy $[1,2,10]$.

The Wiener filter of equation (14) can also be written as :

$$
\psi_{\mathrm{w}}(u, v)=\frac{1}{H(f)}\left[\frac{1}{1+\frac{P_{\mathrm{N}}(u, v) / P_{\mathrm{S}}(u, v)}{|H(f)|^{2}}}\right]
$$

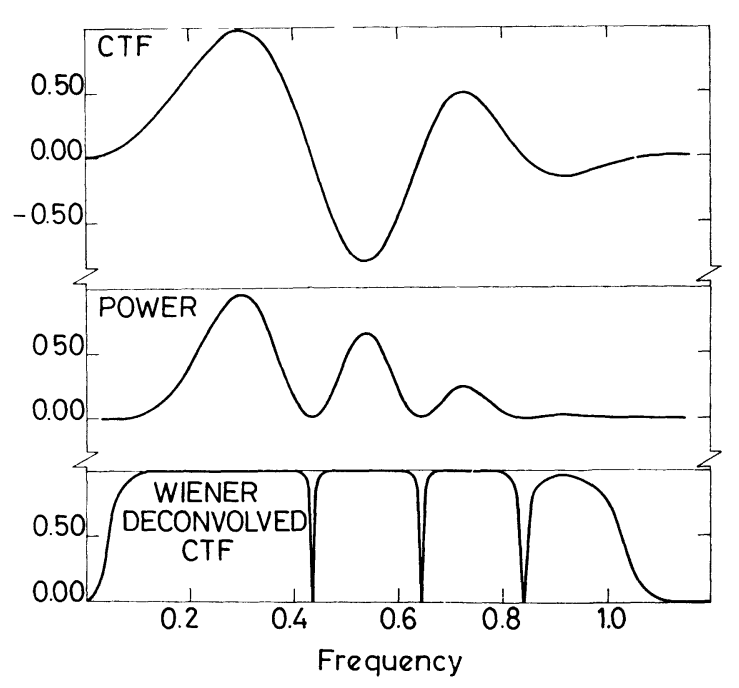

FIG. 1. - Top : The contrast transfer function (CTF) in terms of the generalized frequency

$$
\begin{gathered}
u=\left[\left(C_{\mathrm{s}} \lambda^{3}\right) / 4\right]^{1 / 4} f / \lambda, \\
\operatorname{CTF}(u)=\exp \left(-u^{4} / u_{0}^{4}\right) \sin 2 \pi\left(-u^{4}+D u^{2}\right)
\end{gathered}
$$

with $u_{0}=0.8$ and $D=\Delta z / \sqrt{C_{\mathrm{s}} \lambda}=\sqrt{8}$. Middle : Power spectrum of $\operatorname{CTF}(u)$. Bottom : Wiener filtering of $\operatorname{CTF}(u)$ with

$$
P_{\mathrm{N}} / P_{\mathrm{S}}=10^{-3} \text {. }
$$


where $1 / H(f)$ is known as the inverse filter. Since $P_{\mathrm{S}}$ is not generally known the ratio $P_{\mathrm{N}} / P_{\mathrm{S}}$ is usually taken to be a constant. In fact, one can rewrite the Wiener filter after some manipulation as :

$$
\psi_{\mathrm{w}}(u, v)=\frac{1}{H(f)}\left[1-\frac{P_{\mathrm{N}}(f)}{P_{\mathrm{I}}(u, v)}\right]
$$
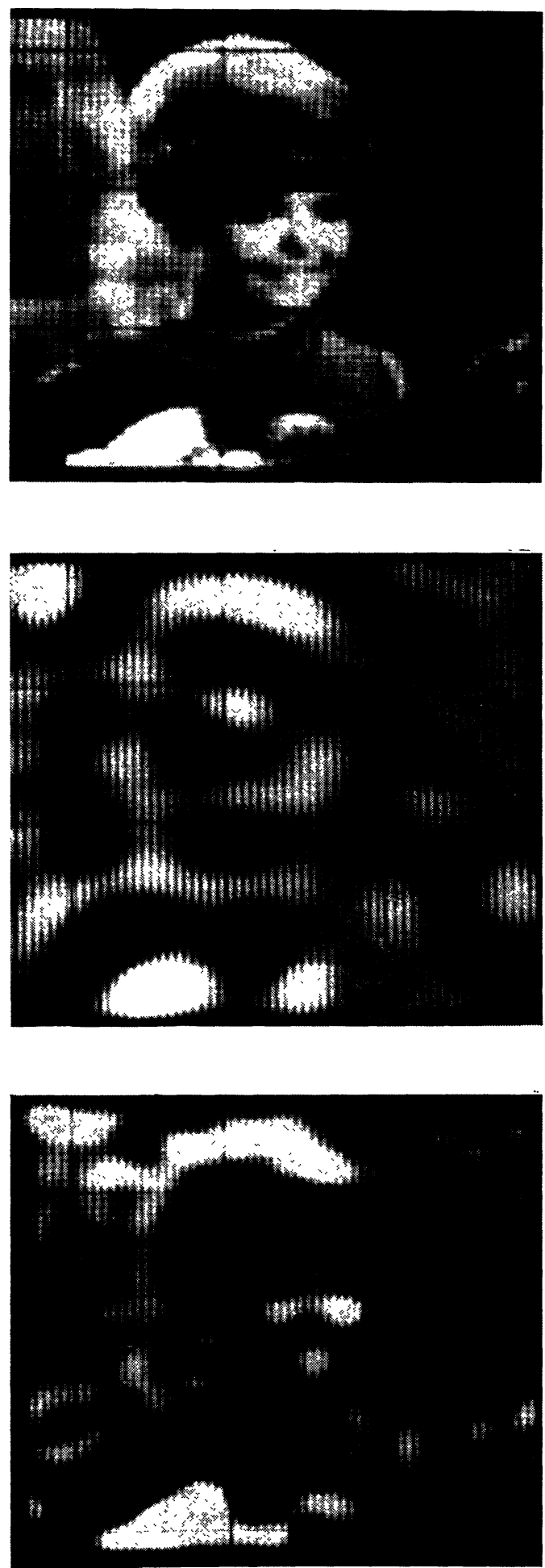

Fig. 2. - Image reduced to $64 \times 64$ elements for display. Top : Original image. Middle : Blurred image with contrast reversal at low frequency and $P_{\mathrm{N}} / P_{\mathrm{S}}=10^{-6}$. Bottom : Restored image after Wiener deconvolution. where $P_{\mathrm{I}}(u, v)$ is the power spectrum of the blurred image, which is always known. From this we realize that the Wiener filter can be exactly calculated, in the case of electron microscopy, with the possible exception of the noise spectrum. It is however more reasonable to substitute $P_{\mathrm{N}}$ by a constant than to do this for $P_{\mathrm{N}} / P_{\mathrm{S}}$ which is obviously not constant.

We have used the actual power spectrum of the image in our restoration.
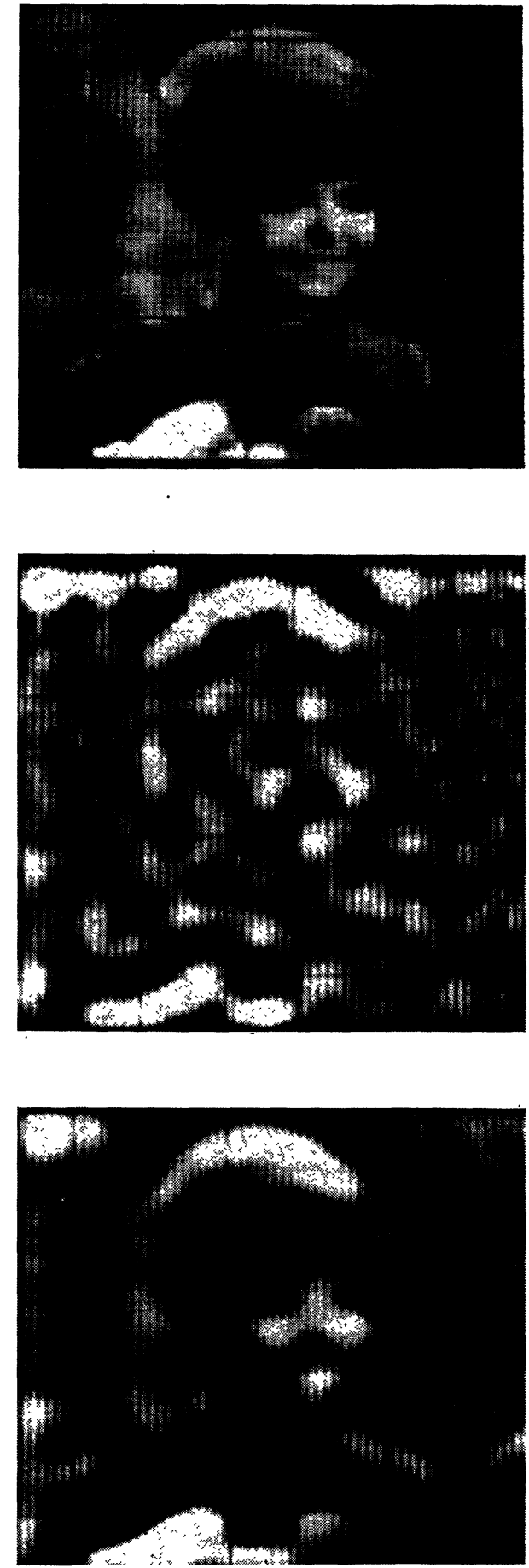

FIG. 3. - Image reduced to $64 \times 64$ elements for display. Top : Original image. Middle : Blurred image with contrast reversal at high frequency and $P_{\mathrm{N}} / P_{\mathrm{S}}=10^{-2}$. Bottom : Restored image after Wiener deconvolution. 
An important property of the Wiener filter is that it does not diverge at the zeros of the transfer function. This is not the case with the inverse filter with which noise can get infinitely amplified at the zeros of $H(f)$.

In connection with the problem of image blurring in electron microscopy the action of the Wiener filter will be to eliminate the contrast reversals that result from the oscillatory character of the transfer function while equalizing the system's response except in those regions where the spectral density of the transfer function is small compared to the ratio $P_{\mathrm{N}} / P_{\mathrm{S}}$. It can be noticed that for spatial frequencies where the spectral density of $H(f)$ is large compared to $\boldsymbol{P}_{\mathrm{N}} / \boldsymbol{P}_{\mathrm{S}}$ the Wiener filter reduces to the inverse filter. To illustrate this we present in figure 1 a characteristic transfer function, its power spectrum and its Wiener filtered correction. The point spread function of transfer functions thus restored has been shown [1] by electron microscopists to produce suitable image restorations.

4. Simulation. - An image of $256 \times 256$ picture elements, each with 256 gray levels, constitutes the reference image (1) with which the simulation was performed. This required first a bidimensional (fast) Fourier transform and multiplication in the frequency domain by a suitable transfer function, then the addition of white noise, and finally a Wiener deconvolution to obtain the restored image. For reasons of simplicity no defocusing was supposed in the transfer function since this assumtion does not alter the basic characteristics of $K(f)$ in equation (6) i. e. an oscillating function with varying period. The transfer function $H(f)$ was parametrized as follows :

$$
H(f)=\exp \left(-f_{0}^{4} / f\right) \sin \left(a f^{4}\right)
$$

(1) Standard image furnished by the Image Processing Institute of the University of Southern California. and the Wiener filter was constructed according to the noise level introduced by a white noise generator. The processing system was a Plurimat S (Intertechnique) adapted for the purpose of image processing. Figures 2 and 3 show the effects of the transfer function on the reference image for different values of the parameters $f_{0}$ and $a$, as well as for different noise levels. In figure 2 the value of $a$ was chosen so that the first zero of $H(f)$ ocurred at a relatively low frequency showing the effect of contrast reversal quite strikingly : i. e. the image is blurred beyond recognition. The restored image, although of poor visual quality, is good enough to permit recognition of human features. Figure 3 is perhaps more typical of weak phase electron microscopy conditions. The first reversal of $H(f)$ ocurrs at a higher frequency and therefore the blurred image retains some rough features of the original. The noise level is also more important on figure 3 than on figure 2. The Wiener filtering is even more satisfactory than in the previous case. This is not an unexpected result : the better the blurred image the better the restoration. In both cases the interest of Wiener filtering seems firmly established.

5. Conclusion. - The application of Wiener filtering techniques to the restoration of images blurred in high resolution electron microscopy is shown in a digital simulation experiment. The results indicate that there is ample justification for such a treatment, digital or optical. The relative value of the two techniques is largely a matter of the equipment available. However, the possibility of implementing different filter structures (i. e. homomorphic deconvolution) which might require nonlinear operations seems to be restricted to digital processing. On the other hand, speed still is on the side of optical computing when this is possible.

\section{References}

[1] Stroke, G. W., Halioua, M., Thom, F. and Willasch, D., Optik 41 (1974) 319.

[2] Burge, R. E. and Scott, R. F., Optik 44 (1976) 159.

[3] LenZ, F. A., Electron Microscopy in Materials Science, Ed. Valdre (Academic Press), 1971, p. 540.

[4] Hanszen, K. J. and Trepte, L., Optik 32 (1971) 519.

[5] Frank, J., Optik 38 (1973) 519.
[6] Helstrom, C. W., J. Opt. Soc. Am. 57 (1967) 297.

[7] Hunt, B. R., Proc. IEEE 63 (1975) 693.

[8] CANnon, M., IEEE Trans. ASSP 24 (1976) 58.

[9] Hunt, B. R., IEEE Trans. on Comp. C-22 (1973) 805.

[10] Srinivasan, V., 1974 S. U. N. Y. Stony Brook, Ph. D. Thesis. 